\title{
EVIDENCIAS DIRECTAS E INDIRECTAS SOBRE LA PROBABLE COEXISTENCIA DE BISONTES Y EL SER HUMANO EN CENTROAMÉRICA DURANTE EL HOLOCENO
}

\author{
Guillermo E. Alvarado ${ }^{1 *}$, Spencer G. Lucas ${ }^{2}$ \& Luis D. Gómez ${ }^{3}$ \\ ${ }^{1}$ Escuela Centroamericana de Geología, Universidad de Costa Rica \\ ${ }^{2}$ New Mexico Museum of Natural History and Science, \\ 1801 Mountain Road NW, Albuquerque, NM 87104-1375 \\ ${ }^{3}$ Organización para Estudios Tropicales, Apdo. 676-2050, \\ San Pedro de Montes de Oca \\ *Autor para contacto: galvaradoi@ice.go.cr
}

(Recibido: 25/10/08 ; aceptado: 12/12/08)

\begin{abstract}
We present a series of paleontological, archaeological and legend-based evidence that suggests to us that the American bison could have coexisted in some refugia with the Amerindians in Central America until as recently as 150 A.D. The aforementioned brings to light the presence of the American bison, the largest extant mammal on the continent, as a possible new source of diet and clothing during the Holocene, which should be considered as a possibility on the list of megamammals that were potentially hunted on the Central American savannahs.

Key words: American bison, archaeology, Holocene, paleontology, Central America.

RESUMEN: Se presentan una serie de evidencias paleontológicas, arqueológicas y de leyendas que nos sugieren que los bisontes americanos pudieron haber coexistido en algunos reductos con los amerindios en América Central en fechas tan tardías como 150 años a.C. Lo anterior trae a la luz la presencia del bisonte americano, el mamífero más grande en la actualidad del continente, como un posible nuevo objeto de alimentación y ropaje durante el Holoceno, que debe de ser considerado como posible dentro de la lista de los megamamíferos que potencialmente eran cazados en las sabanas centroamericanas.

Palabras clave: Bisonte americano, arqueología, Holoceno, paleontología, América Central.
\end{abstract}

ALVARADO, G.E., LUCAS, S.G. \& GOMEZ, L.D., 2008: Evidencias directas e indirectas sobre la probable coexistencia de bisontes y el ser humano en Centroamérica durante el Holoceno.- Rev. Geól. Amér. Central, 39: 53-64. 


\section{INTRODUCCIÓN}

La presencia de fósiles de bisontes en América Central es reconocida en la literatura especializada desde hace varias décadas (Fig. 1). El bisonte fósil (Bison) está reportado en El Salvador (Stirton \& Gealey, 1943, 1949; Webb \& Perrigo, 1984), y en Nicaragua (Williams, 1952; Howell \& Macdonald, 1969; McDonald, 1981; Lucas et al., 2008; Lockley et al., 2008). Para una sinopsis actualizada sobre la paleovertebradología en América Central, ver Lucas et al. (2007).

Sin embargo, igualmente desde hace más de varias décadas, se planteaba la remota posibilidad de que los bisontes pudieran haber existido hasta el Holoceno (Williams, 1952) e inclusive la posibilidad de que sobrevivieran hasta épocas recientes en Centroamérica (ver Gómez, 1986). Efectivamente, recientes estudios en Nicaragua nos traen de nuevo la inquietante posibilidad de la coexistencia del hombre con los bisontes (Williams, 1952; Lockley et al., 2007, 2008), pero mientras que la edad tradicionalmente tomada de unos 6000 años a.P. nos hablaba de su convivencia durante el período arqueológico Arcaico, la nueva edad establecida de tan solo 2100 años a.P. para estratos correlacionarles (ver Schmincke et al., 2009), nos obliga a replantearnos el hecho de que durante el establecimiento de la agricultura y la confección de cerámica precolombina, los bisontes aún poblaban Centroamérica, aunque muy probablemente en forma muy reducida.

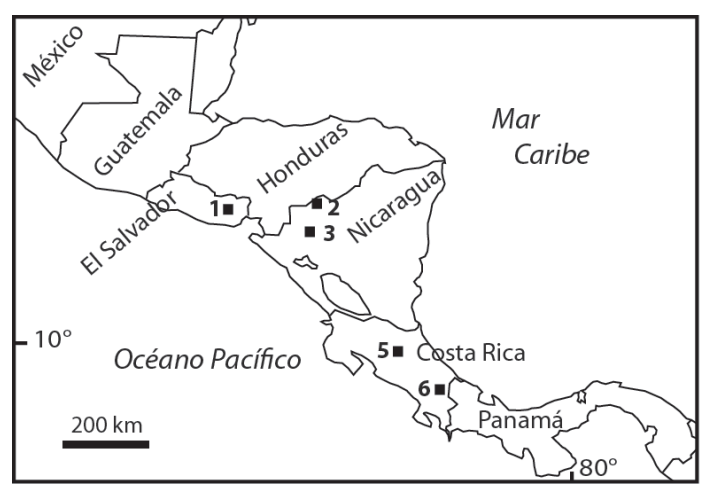

Fig. 1: Principales países y localidades mencionados en el texto: 1. Hormiguero (El Salvador), 2. Jalapa, 3. Río Viejo y El Recreo/El Cauce (Nicaragua), 5. Toro Amarillo y 6. Río Brujo (Costa Rica).
El presente trabajo expone una serie de evidencias sobre la coexistencia de bisontes con el hombre americano en Centroamérica, que varían desde muy sólidas, como lo son la localidad paleontológica El Recreo en Nicaragua, sugestivas como una escultura arqueológica en Costa Rica, y otras menos sólidas como la interpretación de de tradiciones orales -leyendas- en una comarca de Costa Rica.

\section{Los bisontes en el Nuevo y Viejo Mundo}

El Bison emigró de Eurasia a Norte América durante el Pleistoceno, y su presencia más austral en Norte América en la latidud de $55^{\circ} \mathrm{N}$ es usualmente tomado para definir el inicio de la "edad" mamífera-terrestre Rancholabreana (Bell et al., 2004). Asociaciones temporales del arribo del Bison en los 48 estados inferiores de los Estados Unidos de Norteamérica y áreas más al sur están pobremente definidas y tan solo se sugiere una edad de aproximadamente 200 ka (e.g., Kurtén \& Anderson, 1980; Bell et al., 2004). Los bisontes poseen un registro fósil extenso en la parte central y oeste de Los Estados Unidos y sur del Canadá, pero muy escaso en México y América Central.

En Norteamérica existen pocos restos fósiles de bisontes datados más antiguos que 12000 años atrás, pero estos se incrementan significativamente entre 11500 y 7000 años atrás (Fig. 2), lo que al parecer está relacionado con la extinción de otros megamamíferos que se alimentaban del pasto (Mammuthus, Equus, etc.). Entre 7000 y 4000 años atrás, la extensa presencia de bisontes en las grandes praderas al parecer estaba relacionado con la existencia temporal de un clima más cálido (Guthrie, 1990).

La ecología se encuentra, sin embargo, en un gran aprieto cuando ha de justificar por qué los caballos se difundieron durante del Pleistoceno desde el norte hacia el sur de América pero no los bisontes y los berrendos, ungulados herbívoros como los caballos (Simpson, 1983). No obstante, existen reportes aislados de la presencia de bóvidos en Sudamérica: el Colombibos atactodentus en Colombia (Hernández \& Porta, 1960) y el Platatherium en Argentina (Holffstetter, 1971). 

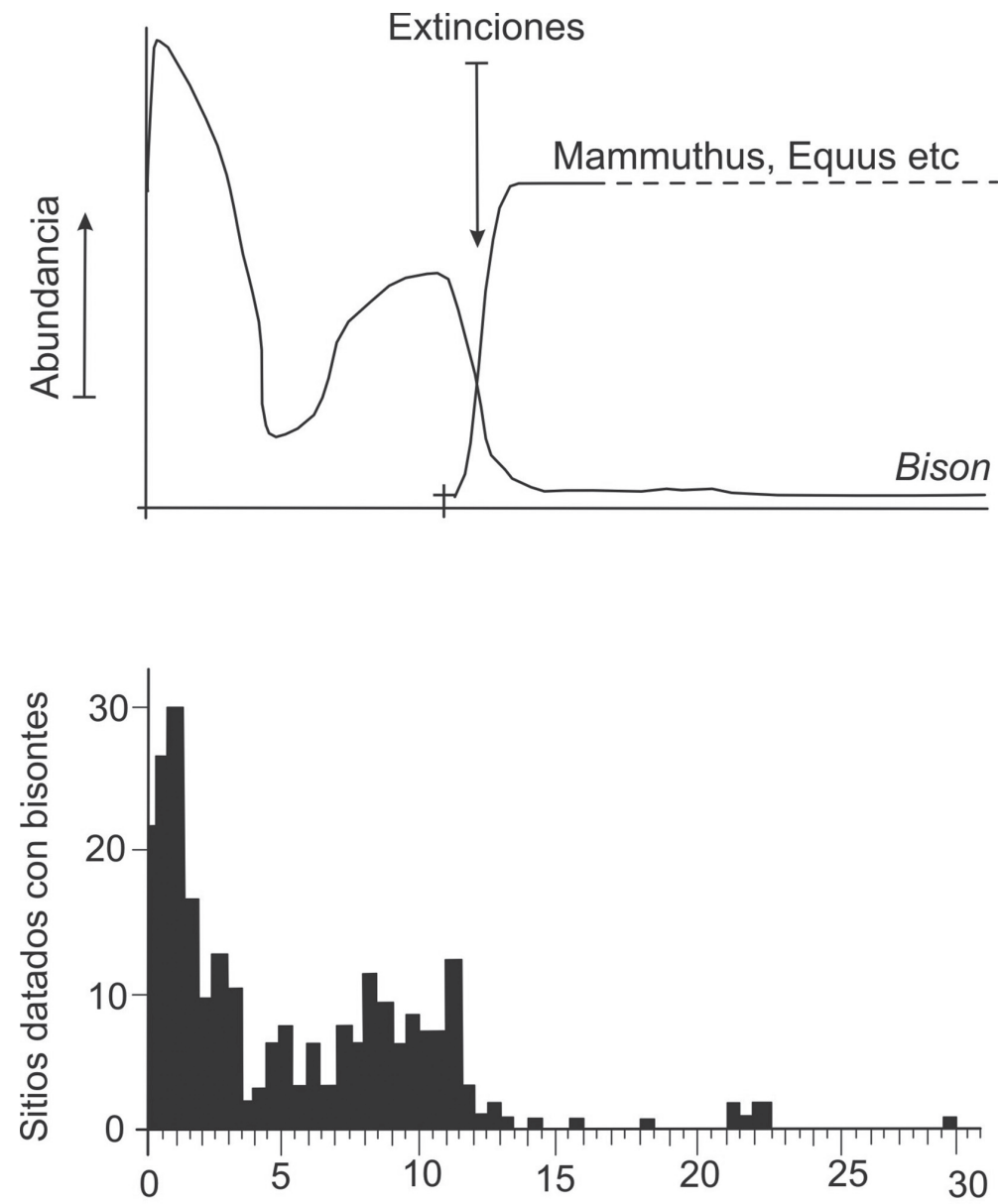

\section{Datos de radiocarbono $\left(\mathrm{C}^{14}\right)$ en miles de años}

Fig. 2: El efecto de la extinción de la megafauna sobre el número de bisontes (simplificado de Guthrie, 1990).

Aunque a priori, la presencia de bóvidos en el Pleistoceno sudamericano parece muy improbable, dado que cuando se implantan en una comarca, suelen vivir en rebaños numerosos, no concordando con los hallazgos aislados y escasísimos restos fósiles, sin embargo, debemos de aceptar su presencia ante las evidencias paleontológicas (Hoffstetter, 1971).

La distribución geográfica de los bisontes durante el Cuaternario era muy extensa (Guthrie, 1990, Fig. 3), pero durante la conquista y colonia fueron muy perseguidos por su carne, pieles y deporte de caza, por lo que casi se le llegó a extinguir en Norteamérica. Hoy día sobrevive en las reservas y parques zoológicos.
Los bisontes son artiodáctilos nómadas, de la familia de los bóvidos y subfamilia de los bovinos. En la actualidad se tienen dos especies de bisontes. El bisonte europeo, Bison bonasus, que sobrevive altamente protegido en pequeños jardines boscosos, en donde prefiere vivir, siendo menos robusto, más alto y delgado que su equivalente norteamericano, el Bison bison, que vive en su estado natural en las praderas de Norteamérica (desde el norte de México hasta Canadá) y es el mayor de todos los mamíferos terrestres existentes en América. Su longitud es de unos $3 \mathrm{~m}$ y su alzada de $1,9 \mathrm{~m}$, siendo más grande los machos que las hembras. Su peso varía entre 450 y $1350 \mathrm{~kg}$, aunque la media es entre 600 y $800 \mathrm{~kg}$. Erróneamente se le ha llamado búfalo americano y 
antiguamente se le conocía como cíbolo, muy abundante al inicio del Holoceno en Norteamérica, pero su caza descontrolada casi lo exterminó a partir del siglo XIX (Il Mondo degli Animali, 1968).

El padre Francisco Ximénez (1722) en su notable Historia Natural del Reino de Guatemala, hace referencia a unos animales llamados "cíbolas", a los que los traductores al español moderno lo interpretan como "búfalos americanos", que correspondería con los bisontes. Ximénez se basó en datos por él recibidos de otras personas, al igual que Francisco López de Gómara, en su Historia general de las Indias de 1552, dedica un capítulo a "Las vacas jorobadas de Quivira" del norte de México, cuya descripción se ajusta a la del bisonte. López de Gómara nunca estuvo en América y de seguro tomó su información de Gonzalo Fernández de Oviedo, quien fue el primer cronista español en mencionar y describir esos artiodáctilos en el Libro 12, capítulo 40, "De las vacas de la tierra septentrional", en la primera parte de su Historia General y Natural de las Indias, de 1535, que no incluyó las ilustraciones que Oviedo indica (cf. Turner, 1985).

De la descripción y re-interpretación de los lugares citados en dichos textos antiguos, se desprende que los primeros bisontes fueron observados en lo que hoy día es el oriente mexicano y muy al norte de México, en la otrora tierra llamada Cíbola o Nueva España, en posible correspondencia con Puebla y alrededores. En efecto, no hay mención de bisontes en el zoológico de Moctezuma en la segunda de las Cartas de Relación de Hernán Cortés a Carlos V, que es muy detallada, así como Bernardino de Sahagún no los menciona en su Historia General de las Cosas de Nueva España (cf. Martín del Campo, 1938-1941).

\section{EVIDENCIAS}

\section{Bisontes Pleistocenos en América Central}

Los registros fósiles confirmados paraAmérica Central son pocos, siendo uno para El Salvador y

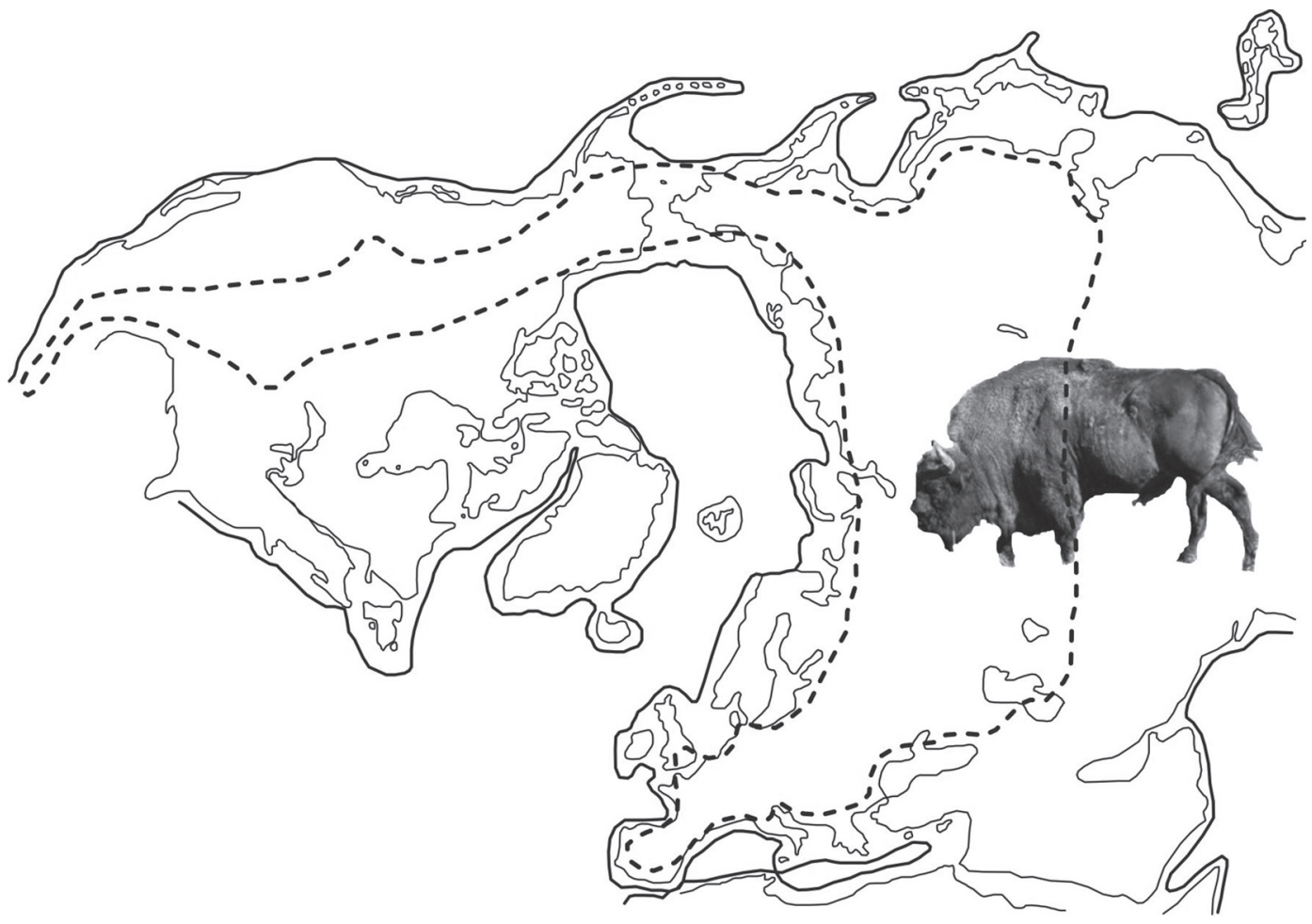

Fig. 3: El gran cinturón de máxima abundancia del bisonte en Eurasia y en Norteamérica y máxima distribución geográfica en Mesoamérica durante el Cuaternario (según Guthrie, 1990, modificada por Alvarado, 1994). 
dos para Nicaragua. Lucas et al. (2007) enlistó el Bison para el Pleistoceno de Honduras, pero este fue un error, dado que ninguna fuente primaria de literatura de los mamíferos hondureños pleistocénicos (McGrew, 1942; Webb \& Perrigo, 1984; Lucas, 2008) lo documentan. El registro de El Salvador está dado para la localidad llamada El Hormiguero (San Juan del Sur), donde el Bison está asociado con el extinto capybara (Neochoerus pinckneyi), gliptodonte (Glyptotherium sp.), el perezoso gigante (Eremotherium laurillardi), tigre dientes de sable (Smilodon sp.), toxodonte (Mixotoxodon larensis) y gonfoterio (Cuvieronious hyodon), todos mamíferos Pleistocénos, así como el caballo (Equus) y camélido (Stirton \& Gealey, 1949; Webb \& Perrigo, 1984; Cisneros, 2008). Un registro Pleistoceno de Bison para el sur de Nicaragua procede de la localidad de Río Viejo, donde está asociado con el toxodonte, el gonfoterio, y el caballo (Howell \& Macdonald, 1969; Lucas et al., 2008). Existe otro registro de Bison para Nicaragua re-examinado por Lucas et al. (2008) según el holotipo de Bos scaphoceras Cope, 1895 para Jalapa en el norte de Nicaragua, para el cual McDonald (1981) dio la sinonimia con la especie del Pleistoceno Tardío B. antiquus. Este registro de Bison está asociado con la fauna de megamamíferos extintos incluyendo gonfoterios, el toxodonte Mixotoxodon y el extinto capibara Neochoerus aesopi (Lucas et al., 2008).

Así, existe un registro fósil Pleistoceno esparcido de Bison que demuestra que el género colonizó Centroamérica (Fig. 1). Tal y como McDonald (1981) denotó, el registro fósil Pleistocénico centroamericano (y del sur de México) de Bison probablemente indica la existencia de pequeñas y aisladas poblaciones de bisontes adaptados al bosque y tierras boscosas. No existen huesos subfósiles Holocenos de Bison hasta ahora encontrados en América Central.

\section{La localidad arqueofaunal de Acahualinca-El Recreo, Nicaragua}

Williams (1952) reporta las huellas de pisadas de bisontes en Nicaragua en el sitio El Recreo. Recientes estudios fueron realizados con alta rigurosidad científica sobre la famosa localidad de
Acahualinca (conocida también como El Cauce) y El Recreo, en las cercanías de Managua, Nicaragua, ya sea desde el punto de vista del análisis de las trazas (pisadas de animales), como desde el punto de vista vulcanológico (Lockley et al., 2007, 2008, 2009; Schmincke et al., 2009).

En la colección de fósiles del Field Museum of Natural History, Chicago (FMNH), dos moldes de las huellas de bisontes catalogadas como FMNH 26998 (Fig. 4) y procedentes de Nicaragua, al parecer provienen con certeza de la localidad El Recreo. En efecto, uno de los moldes (Fig. 4A) calza perfecto con la ilustración de Williams (1952, en su Fig. 11b), y el otro (Fig. 4B) igualmente se parece a la huella ilustrada por Williams (1952, en su Fig. 11e) para El Recreo. Estas son las huellas de un artiodáctilo relativamente grande (hasta $15 \mathrm{~cm}$ de largo y $16 \mathrm{~cm}$ de ancho), cercanamente tan ancho como largo, teniendo un despunte anterior digitiforme, extremos digitiformes, márgenes digitales posteriores redondeadas, y una separación interdigital completa. No se trata de una huella de un camélido por que ellos no tienen un extremo anterior digitiforme divergente, no son puntiagudos anteriormente (ellos carecen de improntas "pinciformes"), además tienen un surco medial dividiendo las impresiones digitales y no son "con forma de corazón" (mucho más ancho posteriormente que anteriormente) siendo todas esta evidencias las que le distinguen de una huella de bóvido de un camélido (Lucas and Hunt, 2007). En su lugar, son muy similares de las huellas de Bison ilustradas por Murie (1974, en su Fig. 130), criterios utilizados para su asignación por Lockley et al. (2008).

Estos estudios trajeron a la luz la existencia de claras huellas de bisontes, zarigüeya (Didelphis) y de unos 15 Homo sapiens (Lockley et al., 2007, 2008, 2009), preservadas en una capa de barro volcánico (ceniza húmeda) en los sitios El Recreo y El Cauce, originalmente consideradas de unos 6500 años de antigüedad, pero que con base en detallados estudios regionales y locales de correlación tefroestratigráfica, geoquímica y radiocronológica, se determinó una edad más reciente de tan solo 2100 años a.P. (Schmincke et al., 2009). Ya Williams (1952) comentaba que la existencia de huellas bisontes en dicho contexto arqueológico, sugería que el bisonte probablemente se extinguió 
en esta región no más tarde que los 2000 años a.P., puesto que nunca se han encontrado huesos asociados con la cerámica precolombina.

Las trazas de bisontes estaban preservadas en el sitio El Recreo (Figs. 1 y 4), 2,5 km al sur del sitio de Acahualinca (El Cauce), pero hoy día no son accesibles, sin embargo, su comprobación puedo realizarse debido al análisis de fotografías y moldes preservados en el Chicago Field Museum de Historia Natural (Lockley et al., 2008). Williams (1952, p. 6 y 28) plantea el hecho de que el nivel estratigráfico de las huellas de bisonte (sitio El Recreo) y las huellas humanas (sitio El Cauce), se encuentran aproximadamente en el mismo nivel estratigráfico. Desgraciadamente, el sitio El Recreo, originalmente utilizado para extraer ladrillos de tobas para construcción (unidad II en la correlación de Schmincke et al., 2009), no es más accesible por el momento debido a que se construyó en dicho lugar.

\section{La arqueo-escultura de un bisote, Costa Rica}

Aproximadamente en 1982, uno de los coautores (LDG) mientras exploraba el río Brujo (unos 5-6 km aguas arriba de su intersección con la Interamericana) en la búsqueda de fósiles de plantas reportadas posteriormente por Kessel (1983) y por Gómez (1986), le informaron de una localidad precolombina superficial en la que se halló una escultura precolombina (Figs. 1 y 5).

Arqueológicamente hablando, es escultura antropomorfa, que tiene las manos o pies pegados al vientre en ambos lados, con tendencia estilística a redondear y exagerar las formas, de las llamadas de bulto, y forma una unidad muy característico de la estatuaria sureña, cuya cronología es tardía, Chiriquí (700-1500 d.C.), de distribución muy amplia, desde la costa pacífica sur hasta tierra adentro, desde Palmar, San Vito, e inclusive San Isidro del General (M. Sanchez, com. escrita, 2009).

Si se analiza la forma con otra perspectiva más abierta, se podría argumentar que posee una clara forma de bisonte, sobresaliendo su característica joroba, cuernos curvados hacia atrás y una gran cabeza en proporción con el cuerpo con inclusive una barbilla (Fig. 5, Tabla 1). De la tabla 1 se desprende que sorprendentemente la pieza arqueológica está confeccionada cercana a la escala de un bisonte americano. La roca tallada al parecer se trata de una arenisca de grano medio, rica en cristales procedentes de rocas ígneas, color café-grisáceo oscuro con leves tonos verdosos (visto en una muesca interna expuesta), pero globalmente, posee un claro tono amarillento superficial.

Alvarado (2006), en su estudio sobre la fuentes de la materia prima de la lítica precolombina del actual territorio de Costa Rica, concluye que los objetos líticos arqueológicos, en su mayoría suelen estar localizados en un radio no mayor de $15 \mathrm{~km}$, y generalmente inferior a los $7 \mathrm{~km}$ de la fuente del material, con excepción de ciertos implementos líticos pequeños con valor canjeable o espiritual (p.ej. jades, cuarzos, etc.). Por ello, la pieza y su litología con toda probabilidad deben de proceder de una fuente cercana. En efecto, la roca de que está constituida, arenisca, abunda en los alrededores del sitio del hallazgo.

Otro hecho notorio es el hecho de que en la región sur de Costa Rica así como en otros lugares del país, siempre se ha discutido sobre la existencia de sabanas actuales y pasadas durante el Pleistoceno. Los tipos de vegetación fósil como actual, parecen sugerir sin lugar a dudas la existencia de sabanas hasta nuestros días (Kessel, 1983; Gómez, 1986), siendo este un hábitat idóneo para los bisontes del pasado.

\section{La leyenda del "toro amarillo", Costa Rica}

Existe una antigua tradición oral entre los pobladores de las pintorescas comarcas de Toro Amarillo o Bajos del Toro" (1400 m s. n. m.) y de Colonia Toro (740 m s. n. m), ambas situadas en el valle intermontano del río Toro (antiguamente llamado Toro Amarillo) en la Cordillera volcánica Central de Costa Rica, en donde se habla que a la llegada de los primeros colonizadores observaron un tipo de "toro" diferente al ganado vacuno que estaba siendo introducido por los españoles (Fig. 1):

"Contaba mi padre que el origen del nombre de la población de Toro Amarillo o Bajos del Toro se remonta a principios del siglo pasado siglo [XIX] cuando los primeros habitantes llegaron a esta región que se ubica en un bajo, procedente 


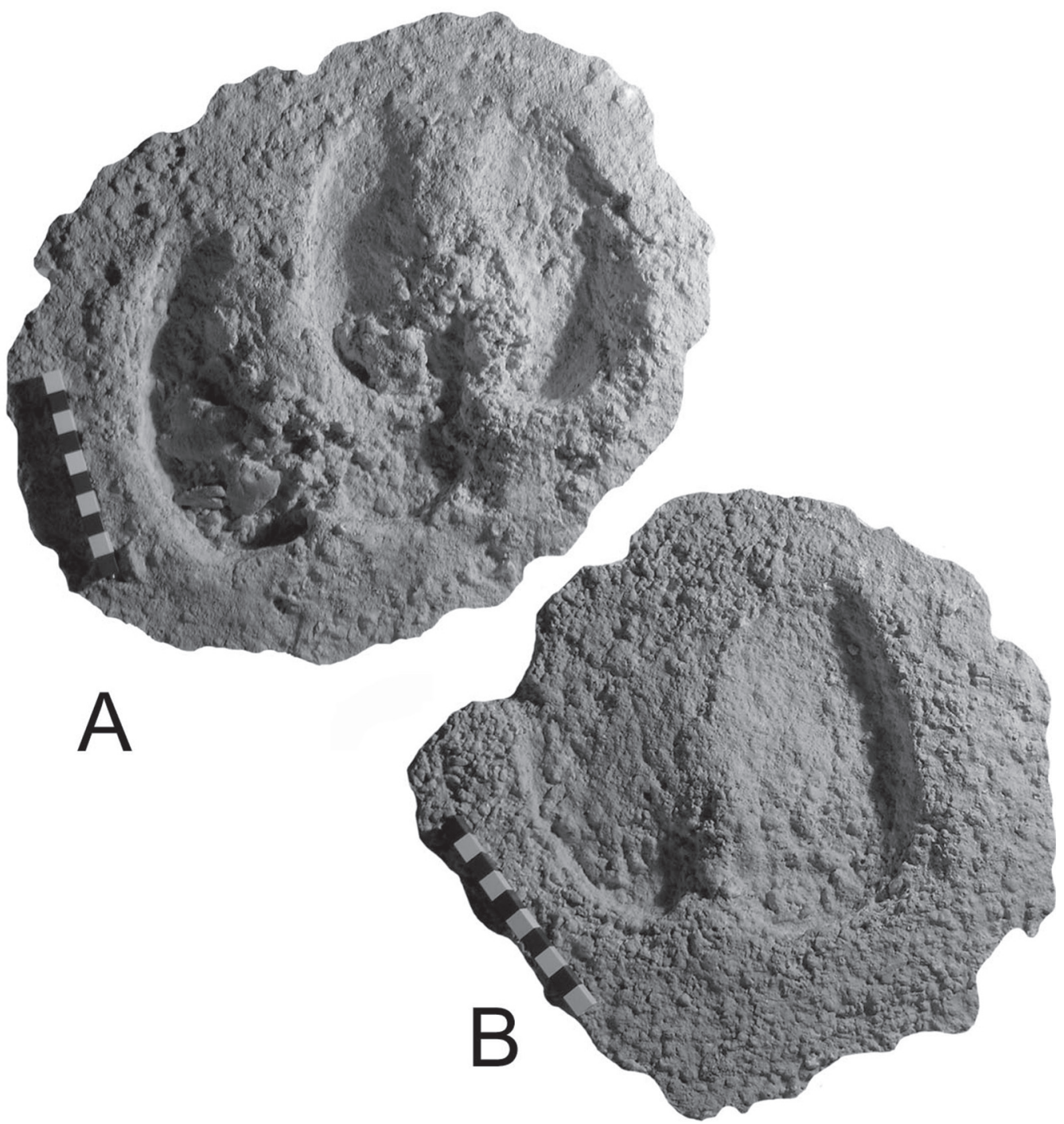

Fig. 4: Moldes de las huellas de Bison del sitio Holoceno El Recreo, Nicaragua, procedentes de la colección del Field Museum of Natural History, Chicago, FMNH 26998. Barra de escala en cm.

de Sabana Redonda, por Poás. Se dice que por aquellos tiempos pastaba un animal semejante a un toro. Su color era amarillento, cuernos vueltos hacia atrás, hocico puntiagudo, mugido fuerte y sus cascos dejaban una marca diferente a la del ganado vacuno. Dicho animal provocaba temor entre los primeros habitantes, muchos de los cuales trataron de darle caza, pero con el tiempo se dieron cuenta que no era peligroso y pastoreaba con el recién introducido ganado vacuno" (O. Porras, com. verb., 1985, en Alvarado, 2000).
Esta leyenda se mantiene aún entre los pobladores ancianos de la región, particularmente en los del distrito de Toro Amarillo del cantón XII de Valverde Vega. provincia Alajuela

Teniendo presente todas las evidencias anteriores en Nicaragua, que son bastante contundentes, la presente leyenda toma fuerza dado que se podría tratar efectivamente de un bisonte, aunque la verdad con probabilidad nunca se llegue a dilucidar. Sin embargo, si resulta sorprendente que dos poblados, un río y un cerro 
Tabla 1

Comparación entre las relaciones de tamaño de cuerpo completo y de la cabeza del bisonte americano con la pieza arqueológica del Brujo.

\begin{tabular}{ccc}
\hline Comparación & Relación largo/alto del cuerpo & Relación alto/largo de la cabeza \\
\hline Bisonte americano & $1,47-1,72$ & $1,22-1,66$ \\
Pieza arqueológica de bisonte & 1,27 & 1,66 \\
\hline
\end{tabular}

(hoy día innominado en los mapas recientes) aparezcan con los nombres Toro (4 veces citado) y con el calificativo de Amarillo (3 veces), demostrando que dicho supuesto rumiante era en efecto particular y diferente al ganado recién introducido. El adjetivo de "amarillo" puede deberse al color que pueden tomar las piedras en el río debido a la acidez de las aguas, tal y como se observó después de las corrientes de barro a raiz del terremoto de Cinchona del 2009, o bien por el color general del rumiante. Resulta muy casual, que el color general de la pieza arqueológica de la zona Sur, previamente descrita, sea de tonos amarillos. El clima del valle del río Toro es tropical lluvioso, pero mientras que en Bajos del Toro las temperaturas suelen ser frescas o incluso frías $\left(16-24^{\circ} \mathrm{C}\right)$, mientras que en la Colonia Toro son algo más cálidas, debido al gradiente topográfico.
En tal caso, las poblaciones de Toro Amarillo (Bajos del Toro) y la Colonia Toro, no es claro cuándo se establecieron, aunque con probabilidad fue en algún momento en el XIX, particularmente Toro Amarillo, puesto que a pesar de que von Frantzius $(1861 \mathrm{a}, \mathrm{b})$ no las menciona en sus trabajos (ya sea por no existir o más probablemente por ser aldeas muy pequeñas), pero sí en cambio menciona el río Toro Amarillo y el cerro del mismo nombre. Ya para 1911 se describe claramente a Toro Amarillo como una población de unas 100 personas distribuidas en 25 casas. No sería de extrañar que este valle estuviese aislado por siglos, debido al profundo cañón fluvial del río Toro, y salvaguardado por los prominentes cerros volcánicos de Chocosuela y Poás, y las temperaturas relativamente gélidas hasta templadas, con una fuerte precipitación pluvial.
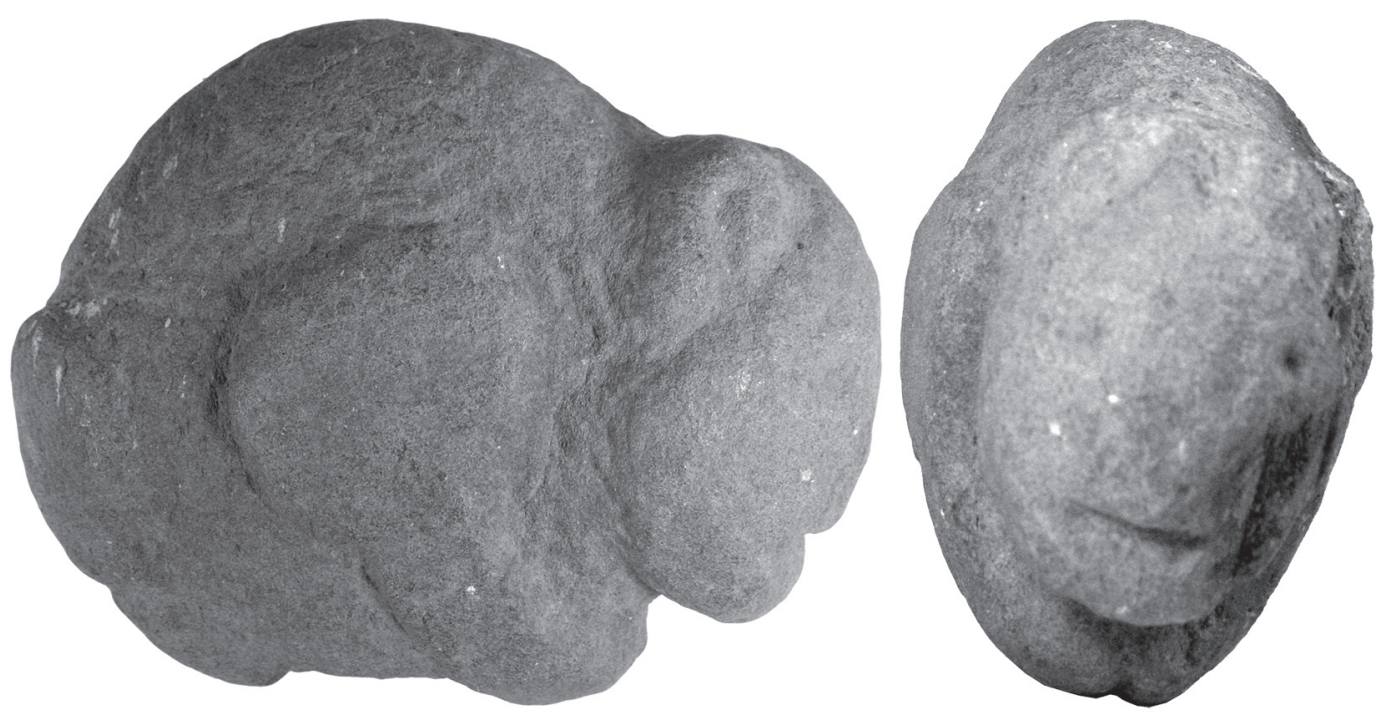

Fig. 5: Figura posible de un bisonte. Vista lateral y frontal. Piedra arenisca. Procedencia: Río Brujo, Costa Rica. Largo: $14 \mathrm{~cm}$. Altura: 11. Período Chiriquí (700-1500 d.C.). Actualmente la pieza forma parte del Museo Nacional de Costa Rica. 


\section{DISCUSIÓN}

La información paleontológica y geocronológica nos indica claramente que los bisontes en Norteamérica se incrementaron significativamente, al contrario que otros megamamíferos, después de la introducción masiva del hombre al nuevo mundo (Guthrie, 1990).

Todas las líneas de evidencia planteadas, desde los inequívocos hallazgos arqueofaunales en Nicaragua, de edad probable de hace unos 2100 años a.P. (aproximadamente 150 a.C.), hasta la escultura zoomórfica en Costa Rica que se puede asociar a un bisonte, nos hace pensar en la convivencia de bisontes con el hombre durante los períodos arqueológicos de Integración Cacical (200 a.C.-1500 d.C.), en donde la pesca y la cacería fueron de alguna importancia, pero sobresalió la agricultura. El que nuestros indígenas describieran oralmente o en su lítica y cerámica faunas lejanas no es frecuente, pero al parecer tampoco algo raro. En Costa Rica se ha descrito una cerámica zoomorfa que representa un típico camélido andino. A ello le debemos de agregar, con su grado de reservas, la interpretación de la débil pero no menos inquietante leyenda de la población bautizada Toro AmarilloBajos del Toro. Todo ello nos obliga a pensar que los bisontes se extendieron desde Norteamérica hasta Nicaragua, y quizás Costa Rica, durante el Holoceno, incluyendo la posibilidad por evaluarse del contacto con los españoles.

La causa de extinción del bisonte americano en Mesoamérica al parecer pudo ser, en sus inicios, las grandes cacerías, pero después el aislamiento hasta su completa extinción desde el sur de México hasta Costa Rica, al estar representados por poblaciones aisladas en lugar de grupos extensos dispersos en grandes praderas, particularmente al tener contacto con los humanos.

Todo esto nos abre una nueva página en la cual un megamamífero, hoy extinto en Mesoamérica, pudo ser objeto de alimentación y ropaje durante el final del Pleistoceno (Fig. 6) y gran parte del Holoceno y, por ello, debe de ser agregado a la lista probable de fauna que cazaban los amerindios en Centroamérica y deberá de re-evaluarse con otros ojos algunas de las figuras precolombinas antropomórficas y zoomórficas a la luz de estos resultados. Claramente, nuevos estudios paleotológicos, tefroestratigráficos y geoarqueológicos son requeridos.

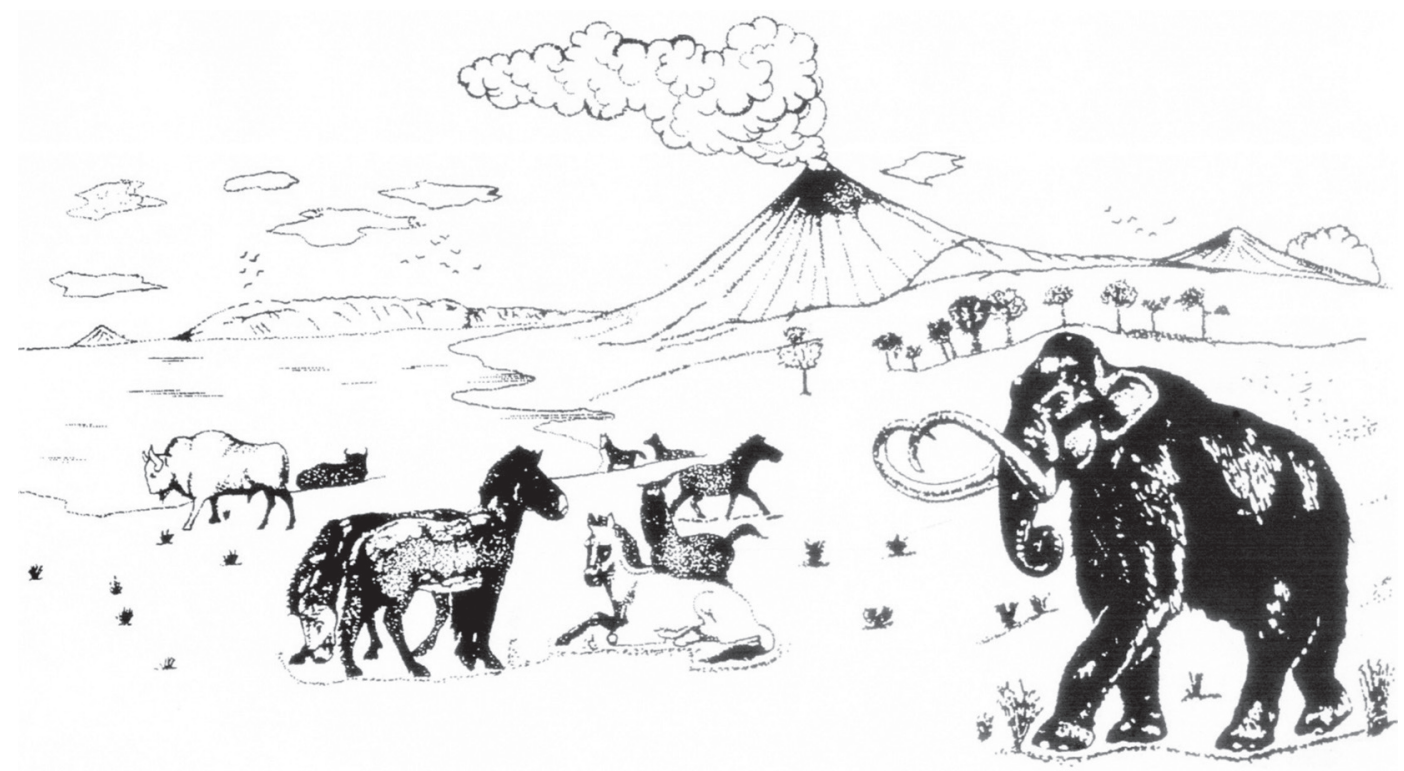

Fig. 6: Reconstrucción de una escena del Pleistoceno en una sabana y sabana arbolada en el lago de Nicaragua mirando hacia Costa Rica. Se observan los bisontes, caballos y un elefante imperial. Basado en Alvarado (1994). 


\section{AGRADECIMIENTOS}

Se agradece a S. Bonis, M. Sánchez, P. Fernández, W. Pérez, C. Fernández y J. Chaves por la información aportada.

\section{REFERENCIAS}

ALVARADO, G.E., 1994: Historia Natural Antigua: Los intercambios biológicos interamericanos.232 págs. Ed. Tecnológica de Costa Rica.

ALVARADO, G.E., 2000: Los volcanes de Costa Rica: Su geología, historia y riqueza natural [ $2^{\mathrm{a}}$ ed.]. $-\mathrm{xv}+269$ págs. EUNED, San José.

ALVARADO, G.E., 2006: Objetos líticos precolombinos en Costa Rica: Tipos, frecuencia petrográfica, materia prima y aspectos sobre su utilización. Pre-Columbian Lithic Objects in Costa Rica: Types, Petrographic Frequency, Raw Materials, and Aspects of Use.- En: FERNÁNDEZ, P. \& ALVARADO, G.E. (eds.): Artesanos y piedras. Herramientas y escultura precolombina en Costa Rica. Craftsmen \& stones. Pre-Columbian stone tools and sculpture in Costa Rica. Fundación Museos del Banco Central: 71-127.

BELL, C.J., LUNDELIUS, E.L., BARNOSKY, A.D., GRAHAM, R.W., LINDSAY, E.H., RUEZ, D.R., SEMKEN, H.A., WEBB, S.D., ZAKREWSKI, R J., 2004. The Blancan, Irvingtonian, and Rancholabrean mammal ages.- En: WOODBURNE, M.O. (ed.): Late Cretaceous and Cenozoic mammals of North America: Biostratigraphy and geochronology. New York, Columbia University Press: 232-314.
CISNEROS, J.C., 2008: The fossil mammals of El Salvador.- En: LUCAS et al. (ed.): Neogene Mammals, New Mexico Museum of Natural History and Science, Bulletin, 44: 375-380.

COPE, E.D., 1895: Extinct Bovidae, canidae, and felidae from the Pleistocene of the Plains.- Journal of the academy of natural sciences of Philadelphia, series 2, 9: 453-459.

GÓMEZ, L.D., 1986: Vegetación de Costa Rica.327 págs. EUNED, San José.

GUTHRIE, R.D., 1990: Frozen fauna of the Mammoth Steppe: the history of Blue Baby.- xiv +323 págs. The University Chicago Press.

HERNÁNDEZ J. \& PORTA, J. DE, 1960: Un nuevo Bóvido pleistocénico de Colombia: Colombibos atactedentus.- Bol. Geol., Bucaramanga, 5: 41-52.

HOFFSTETER, R., 1971: Los vertebrados Cenozoicos de Colombia: Yacimientos, faunas, problemas planteados.- Geol. Colombiana, 8: 37-61.

HOWELL, T.R. \& MACDONALD, J.R., 1969: A Pleistocene vertebrate fauna from Nicaragua.- Geol. Soc. Amer., Special Paper 212: 143-144.

KESSEL, R.H., 1983: Quaternary history of the Río General Valley, Costa Rica.- Nat. Geogr. Soc. Res. Rep., 15: 339-358.

KURTÉN, B. \& ANDERSON, E. 1980: Pleistocene mammals of North America.443 págs. Columbia University Press, New York. 
LOCKLEY, M.G., GARCIA-VASQUEZ, R., ESPINOZA, E. \& LUCAS, S.G., 2007: Notes on a famous but "forgotten" human footprint site from the Holocene of Nicaragua.- New Mexico Museum of Natural History and Science Bulletin, 42: 97-102.

LOCKLEY, M.G., LUCAS, S.G., HUNT, A.P., GARCIA-VASQUEZ \& R., ESPINOZA, E., 2008: Non hominid vertebrate traces from the lower Holocene of Nicaragua.New Mexico Museum of Natural History and Science Bulletin, 44: 393-397.

LOCKLEY, M.G., GARCIA VASQUEZ, R., ESPINOZA, E. \& LUCAS, S.G., 2009: America's most famous human footprints: History, context and first description of mid-Holocene tracks from the shores of Lake Managua, Nicaragua.Ichnos, 16: 55-69.

LUCAS, S.G. 2008: Pleistocene mammals from Yeroconte, Honduras.- En: LUCAS et al. (eds.): Neogene Mammals, New Mexico Museum of Natural History and Science.Bull. 44: 403-407.

LUCAS, S.G. \& HUNT, A.P., 2007: Ichnotaxonomy of camel footprints.- New Mexico Museum of Natural History and Science Bulletin 42: 155-168.

LUCAS, S.G., ALVARADO, G.E., GARCÍA, R., ESPINOZA, E., CISNEROS, J.C. \& MARTENS, U., 2007: Vertebrate paleontology.- En: BUNDSCHUH, J. \& ALVARADO, G.E. (eds.): Central America: Geology, Resources and Hazards. Taylor \& Francis, Londres/ London. 1: 443-451.
LUCAS, S.G., GARCIA, R., ESPINOZA, E., ALVARADO, G.E., HURTADO DE MENDOZA, L. \& VEGA, E., 2008: The fosil mammals of Nicaragua.- En: LUCAS et al. (eds.): Neogene Mammals, New Mexico Museum of Natural History and Science Bull. 44: 417-429.

MARÍN DEL CAMPO, R., 1938-1941: Ensayo de interpretación del Libro undécimo de la Historia General de las Cosas de Nueva España de fr. B. de Sahagún.- Anales Inst. Biología 9: 379-391. 1938; 11: 385-408. 1940; 12: 489-506. 1941.

MCDONALD, J. N., 1981: North American bison: Their classification and evolution.- 316 págs., Berkeley, University of California Press.

MCGREW, P.O. 1942: Field Museum paleontological expedition to Honduras.- Science, 96: 85 .

MURIE, O.J. 1974: A field guide to animal tracks.375 págs. Houghton Mifflin Co., Boston.

SCHMINCKE, H.U., KUTTEROLF, S., PÉREZ, W., RAUSCH, J., FREUNDT, A. \& STRAUCH, W., 2009: Walking through volcanic mud: 2,100-year-old Acahualinca footprints (Nicaragua) I: Stratigraphy, lithology, volcanology and age of the Acahualinca section.- Bull. Volcanology. 71:479-493. DOI 10.1007/ s00531-009-0438-0

RIZZOLI EDITORE, 1968: Il Mondo degli Animali. Trad. española: El mundo de los animals.- Ed. Noguer, S.A., vol. I: fascículos: 1, 5; vol. II: fascículos 22,23, 37 y 44; vol. III: fasc. 50; Barcelona. 
SIMPSON, G.G., 1983: Fossils and the History of Life. Trad. española: Fósiles e historia de la vida.- 240 págs. Ed. Labor, Bibl. Scient. Am., España.

STIRTON, R.A. \& GERLEY, W.K., 1943: Fosil vertebrates from El Salvador.- J. Paleontology, 17: 309.

STIRTON, R.A. \& GEALEY, W.K., 1949: Reconnaisance geology and vertebrate paleontology of El Salvador, Central America.- Bull. Geological Soc. Amer., 60: 1731-1754.

TURNER, D. 1985: Forgotten treasures from the Indies: The illustrations and drawings of Fernández de Oviedo.- Huntington Library Quarterly 48: 1-46. 1985.

VON FRATZIUS, A., 1861a: Beiträge zur Kenntniss der Vulkane Costa Rica's. Trad.
Española: Aporte al conocimiento de los volcanes de Costa Rica.- En: C.A. Vargas (ed., 1979): 11-32.

VON FRANTZIUS, A., 1861b: Originalkarte des nördlichen Theiles von Costa Rica.- Gotha, Justus Perthes.

WEBB, S.D. \& PERRIGO, S., 1984: Late Cenozoic vertebrates from Honduras and El Salvador.- J. Vertebrate Paleontology, 4(2): $237-264$.

WILLIAMS, H., 1952: Geological observations on the ancient human footprints near Managua.- Contributions to American Anthropology and History, 52: 1-31.

XIMÉNEZ, F., 1772: Historia Natural del Reino de Guatemala.- 351 págs. Obra introducida y cotejada por E. Chinchilla, J.R. Herrera y F. Gall, 1967, Ed. "José de Pineda Ibarra". 